\title{
El aprendizaje-servicio como aporte a la formación profesional: un análisis de caso a partir de la metodología Quinta Dimensión
}

\section{Service Learning as a Contribution to Professional Training: f Case Analysis Based on the fifth Dimension methodology \\ f aprendizagem-serviço como aporte à formação profissional: uma análise de caso a partir da metodologia Quinta Dimensão}

\author{
José Eduardo Sánchez Reyes* \\ Universidad Icesi \\ Yamileth Bolaños Martínez** \\ Universidad Icesi
}

Doi: http://dx.doi.org/10.12804/revistas.urosario.edu.co/apl/a.8197

\section{Resumen}

En este artículo se describe el proceso de participación de estudiantes universitarios de psicología en la adaptación e implementación de un modelo educativo 5D denominado proyecto Mohán. El modelo se asume como experiencia de aprendizaje-servicio, y se explora cómo esta metodología aporta a la comprensión del oficio del psicólogo. En la experiencia participaron 8 estudiantes universitarios, 1 profesor y 1 estudiante de maestría. La metodología de investigación empleada es un experimento por diseño. Los resultados permiten plantear que la experiencia de participar en el desarrollo de una intervención directa, como una situación de aprendizaje, posibilita que los estudiantes asuman un rol de psicólogos, además de desarrollar aprendizajes del oficio y competencias profesionales.
Palabras clave: psicología cultural, aprendizaje servicio, comunidad de práctica.

\section{flbstract}

This article describes the involvement process of psychology university students in the adaptation and implementation of a 5D model known as Proyecto Mohán. The research assumed a service-learning experience and explored how this methodology contributed to the understanding of the psychologist's theory and profession. The experience involved eight university students, one professor, and one master's student. The research methodology used is the experiment by design. Based on the results, we argue that the experience of participating in the development of a direct intervention as a learning situation allows students to assume the role

\footnotetext{
* Dirigir correspondencia a José Eduardo Sánchez Reyes, Correo electrónico: jesanchez@icesi.edu.co ORCID: https://orcid. org/0000-0002-4580-1894

** ORCID: http://orcid.org/0000-0003-0863-6559

Para citar este artículo: Sánchez Reyes, J. E., \& Bolaños Martínez, Y. (2019). El aprendizaje-servicio como aporte a la formación profesional: un análisis de caso a partir de la metodología Quinta Dimensión. Avances en Psicología Latinoamericana, 37(3), 489-504. Doi: http://dx.doi.org/10.12804/revistas.urosario.edu.co/apl/a.8197
} 
of psychologists, in addition to developing apprenticeships in the profession and professional competencies. Keywords: Cultural psychology, service learning, community of practice.

\section{Resumo}

Neste artigo descreve-se o processo de participação de estudantes universitários de psicologia na adaptação e implementação de um modelo educativo denominado projeto Mohán. O modelo assume-se como experiência de aprendizagem-serviço e se explora como esta metodologia aporta à compreensão do ofício do psicólogo. Na experiência participaram 8 estudantes universitários, 1 professor e 1 estudante de mestrado. A metodologia de pesquisa empregada é um experimento por desenho. Os resultados permitem apresentar que a experiência de participar no desenvolvimento de uma intervenção direta como uma situação de aprendizagem permite que os estudantes assumam um papel de psicólogos, para além de desenvolver aprendizagens do ofício e competências profissionais.

Palavras-chave: psicologia cultural, aprendizagem-serviço, comunidade de prática.

Este siglo es escenario de problemáticas como la pobreza, la violencia, la globalización económica, el cambio climático, la crisis ambiental y la creciente desigualdad. En ese escenario, surgen los Objetivos de Desarrollo Sostenible (ODS), trazados por las Naciones Unidas (2016), en ellos se considera la educación como uno de los principales factores de desarrollo, siendo la universidad un "agente de cambio, catalizador de la acción política y social" llamada a cumplir un papel importante en el mejoramiento de esas problemáticas.

En este sentido, se ha señalado que la formación de profesionales requiere nuevas maneras de enseñar y aprender, que doten a los estudiantes de capacidad de análisis, autonomía en el aprendizaje, disposición al trabajo cooperativo, además de aplicabilidad de conocimientos teóricos que permitan su uso en contextos reales (Pozo \& Monereo, 2009). Lo anterior establece retos en la formación de futuros profesionales que posean conciencia social, habilidades investigativas y de intervención para enfrentar las diferentes demandas de una sociedad mundial en evolución (Gómez-Estern, Martínez-Lozano \& Vásquez, 2014).

Por lo tanto, este artículo describe cómo la metodología de aprendizaje-servicio aporta a la comprensión de la teoría y del oficio de un grupo de estudiantes universitarios de psicología en un caso específico: la adaptación y desarrollo del modelo Quinta Dimensión (5D) por medio del proyecto Mohán 5D. El modelo 5D es adaptado por el semillero ${ }^{1}$ de investigación de psicología educativa en una universidad privada de Cali, Colombia. En la experiencia participaron ocho estudiantes universitarios, un profesor y un estudiante de maestría durante los años 2016 y 2017. El análisis se centra en los aprendizajes obtenidos por los estudiantes universitarios en el proceso de adaptación y desarrollo del modelo 5D. La metodología de aprendizaje-servicio, de acuerdo con los resultados encontrados, demuestra ser valiosa tanto en el aprendizaje conceptual (García-Romero, Sánchez \& Lalueza, 2018; Gómez-Estern, Martínez-Lozano \& Vásquez 2014; Blanton, Bremme \& Nocon, 2006), como en el desarrollo de competencias del oficio de los estudiantes universitarios como psicólogos, aprendizajes desarrollados y potenciados por medio de la constitución de una comunidad de práctica (Wenger, 2008). Los resultados se presentan haciendo referencia a los productos de la implementación del proyecto Mohán 5D y se retoman las valoraciones que hacen los estudiantes después de haber concluido la experiencia.

\footnotetext{
Espacios dedicados a la adquisición de herramientas de investigación en un campo específico, en los que participan estudiantes orientados por uno o varios docentes con conocimientos en el área.
} 


\section{Modelo educativo Quinta Dimensión}

Michael Cole (1999) y el Laboratory of Comparative Human Cogniton (LCHC) desarrollaron un modelo de investigación e inclusión educativa denominado Quinta Dimensión (5D), que entiende el aprendizaje como un proceso social y producto de la interacción mediada por artefactos culturales, siendo la colaboración un elemento facilitador (Cole, 1999).

La 5D, inspirada en la psicología histórico cultural, es un sistema de actividades que busca potenciar el desarrollo humano, por medio de interacciones mediadas que posibilitan la configuración de diversas Zonas de Desarrollo Próximo (ZDP) (Cole, 1999; Baquero, 1996). Se configura mediante sus propias reglas, roles sociales, artefactos y entorno ecológico, además de contar con principios de implementación como el énfasis en el contexto, la participación voluntaria de los niños y niñas, la creación de actividades lúdico-educativas atractivas para los participantes, y la generación de condiciones para la creación de ZDP. Adicionalmente, resulta central en la 5D la existencia de una narrativa alrededor de un personaje mágico que conserva el bienestar del sistema y mantiene una comunicación constante con los niños con la ayuda de cartas (Cole, 1999, 2017).

Así, la 5D permite alcanzar el aprendizaje significativo, por medio del cual cada sujeto se "re-posiciona" en los interrogantes de su cultura, al acceder a las respuestas que sus predecesores han elaborado y elaborando las suyas (Meireu, 1998; citado en Sánchez, 2018).

\section{Transformaciones de los estudiantes por medio del Aprendizaje servicio y la 5D}

En la implementación reciente de la 5D en entornos escolarizados se ha planteado que es necesario un mayor análisis de los roles de los estudiantes universitarios que participan en el modelo, no solo como instrumentos de cambio sino como agentes que aprenden y cuya identidad puede cambiar cuando participan de actividades de investigación-intervención como la 5D (Lamas, Sánchez-Busqués \& Lalueza, 2018).

Los cambios en el desarrollo propiciados por parte de la 5D se sustentan en la perspectiva ecológica de Urie Bronfenbrenner y perspectivas socioculturales del desarrollo como las de la psicología cultural de Vygotski (1993). Específicamente, Bronfenbrenner plantea que el desarrollo humano acontece en contextos de variados niveles de intercambio mutuo e inclusión (Cole, 2016), en donde los participantes cambian de roles, de entorno o los dos a la vez (Bronfenbrenner, 1991).

En esta vía, la participación de estudiantes universitarios en la 5D ha potencializado su aprendizaje por medio de pedagogías innovadoras, que les permiten resolver problemas por medio de la conexión entre la teoría y las situaciones de la vida real (Gómez-Estern, Martínez-Lozano \& Vásquez, 2014).

Blanton, Bremme y Nocon (2006), en sus experiencias de implementación del modelo 5D en EE. UU., muestran transformaciones en la apropiación de conceptos como aprendizaje, enseñanza y cultura por parte de estudiantes universitarios, así como un incremento de su capacidad para usar herramientas conceptuales asociadas con prácticas profesionales.

Por su parte, Lalueza, Sánchez-Busqués y Padrós (2016) establecen que la implementación del proyecto Shere Rom en Barcelona (adaptación del modelo 5D) es una experiencia de aprendizaje-servicio, en la que la universidad crea vínculos estrechos con la comunidad, vínculos que expresan dos aspectos fundamentales para este tipo de relación: se ofrecen respuestas a problemáticas reales - lo que supone un retorno social de la investigación-y los estudiantes participantes adquieren conocimientos y desarrollan competencias de su currículo mediante una experiencia real.

El aprendizaje-servicio puede considerarse como parte de una diversa tradición de pensamiento, 
en donde la actividad tiene un importante lugar como unidad de análisis (Fardella \& Carvajal, 2018). Una de estas primeras perspectivas en torno a la práctica es la de Jhon Dewey, de acuerdo con la cual el aprendizaje impuesto por la escolarización basado en la verbalización y la abstracción debería ser reemplazado por:

[...] una escuela donde la actividad constructiva, práctica e intelectual será el centro y la fuente de todo, y partiendo de esta idea de trabajo se desarrollará siempre en dos direcciones: por un lado, las implicaciones sociales de esta actividad constructiva, y por otro, el contacto con la naturaleza que facilita los medios materiales [...]. (Dewey, 1894; en Menand, 2001, p. 302)

En consecuencia, Dewey propone considerar la educación esencialmente como escenario de construcción de experiencias, en donde el hombre se entiende como experimentador activo que dota de sentido a la experiencia y logra unir la teoría y la praxis (Fardella \& Carvajal, 2018).

\section{Método}

La metodología empleada se denomina 'experimento por diseño'. Esta metodología permite estudiar cómo los procesos de transformación del entorno social potencian el desarrollo humano, ha resurgido en las últimas décadas y ha tomado diversos nombres, entre los cuales se menciona la intervención formativa y la experimentación de diseño social (Cole, 2016).

Las anteriores denominaciones comparten, dentro de sus características, la necesidad de transformar las circunstancias educativas en conjunto con la comunidad en donde tienen lugar: el estudio del aprendizaje en la cotidianidad, el entendimiento del aprendizaje desde una perspectiva ecológica, además de compartir enfoques teóricos que se preguntan por el aprendizaje en contexto (Gutiérrez \& Jurow, 2016).
Cole (2014) propone la necesidad de rastrear el ciclo de vida del diseño e implementación de una actividad que tuvo como objetivo general potenciar el desarrollo humano y ubica al modelo de la 5D dentro de este enfoque metodológico de experimento por diseño.

La adaptación del modelo 5D, denominado 'Proyecto Mohán 5D', tuvo esencialmente dos planos de intervención, en el primer plano se ubican los niños y niñas participantes del proyecto, los agentes educativos de la ludoteca y los estudiantes universitarios, y en el segundo, los estudiantes universitarios y los profesores. En cada uno de estos planos de interacción se crearon diversas Zonas de Desarrollo Próximo (ZDP) (Baquero, 1996). Este artículo se enfocará en el análisis de los cambios en las habilidades de los estudiantes universitarios participantes en el proyecto.

\section{Procedimiento}

El proyecto 5D fue adaptado en una ludoteca ubicada en un barrio en la ciudad de Cali, Colombia. El interés de la adaptación del modelo 5D, denominado 'Mohán', por una parte, era propiciar las habilidades de investigación e intervención con sentido social en los estudiantes participantes del semillero de psicología educativa, que les permitiera tener un repertorio mayor de habilidades al momento de realizar su práctica profesional. Por otra parte, buscaba conocer las necesidades educativas del contexto, para adaptar el proyecto mencionado como prueba piloto y establecer un vínculo de trabajo en conjunto entre la universidad y la comunidad que asistía al espacio. ${ }^{2}$ Este artículo se enfoca en el análisis del primer interés relacionado con el desarrollo de competencias profesionales en los estudiantes de psicología.

Actualmente, el trabajo conjunto entre la universidad y la comunidad de la Ludoteca continúa con una nueva propuesta del modelo 5D. 
Las actividades del Semillero de Psicología Educativa se desarrollaron en los años 2016 y 2017. Durante el primer semestre de 2016 el semillero fue convocado por dos docentes de este campo con conocimiento y experiencia en la implementación del modelo 5D. El semillero se constituyó como un espacio de asistencia voluntaria y extracurricular para los estudiantes.

En el segundo semestre de 2016 el semillero de investigación, ya conformado, adoptó una metodología tipo seminario. Las reuniones del semillero se realizaban con frecuencia semanal. Durante el primer semestre de 2017, los integrantes del semillero conocían teóricamente el modelo y establecieron contacto con una Ludoteca en un barrio de la ciudad de Cali. Se llevaron a cabo tres visitas preliminares al espacio, durante las cuales se entablaron conversaciones con las directivas del lugar, docentes de básica primaria y niños y niñas que asistían a esta, para conocer qué necesidades educativas señalaba la comunidad e iniciar un diálogo entre la universidad y la comunidad que permitiera establecer un plan de trabajo conjunto.

De forma paralela, durante el primer semestre de 2017, el semillero se reunía dos veces por semana para construir una narrativa alrededor de un personaje mágico relacionado con las tradiciones del sector, surgió el nombre de 'Mohán'. Posteriormente, las reuniones se enfocaron en planear y ajustar colaborativamente las actividades de la
5D que se llevaron a cabo en la Ludoteca. Cada reunión contó con un acta que resumía los temas y tareas acordadas.

La implementación de la 5D se llevó a cabo en el segundo semestre de 2017. Las actividades seguían presupuestos básicos de la 5D propuestos por Cole (1999): permitían intercambios intergeneracionales en donde los roles podían variar, había un personaje mágico (Mohán) que mediaba en las actividades, por medio de la escritura de cartas dirigidas a los niños y niñas participantes. Respecto a las actividades, se buscaba que los niños y niñas quisieran participar voluntariamente en ellas, cada actividad tenía una guía que podía ser leída por quienes participaban, con ayuda de los estudiantes universitarios, y se desarrollaban en grupos conformados por uno estudiante universitario y tres o cuatro niños. Las actividades, de acuerdo con el diálogo entre comunidad y universidad, se centraron en el mejoramiento de habilidades matemáticas y de lectoescritura de los participantes (figura 1).

Al finalizar la experiencia de implementación, se hicieron tres jornadas de recuperación y evaluación de la experiencia, posteriores a su realización. Estas jornadas implicaron la realización de grupos focales y entrevistas semiestructuradas con los integrantes del semillero sobre su participación en la experiencia, se tuvieron en cuenta tres macrocategorías: vinculación al semillero, roles al interior del semillero y posibilidades de la 5D.

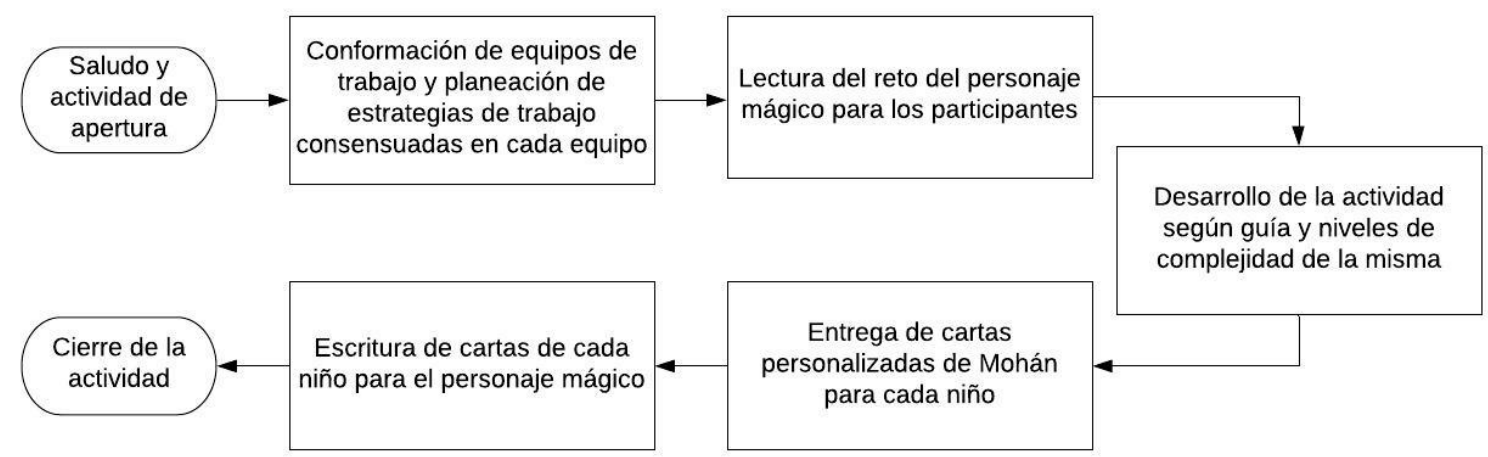

Figura 1. Orden de las sesiones del Proyecto Mohán 5D 
Durante los espacios mencionados, se retomaron registros fotográficos, actas de reunión del semillero, guías de actividad, documentos de caracterización, diarios de campo, además de cartas hechas por los niños y por el semillero mediante el personaje mágico Mohán.

\section{Participantes}

En esta investigación participaron 8 estudiantes universitarios, 1 docente y 1 estudiante de maestría, quienes realizaron el diseño e implementación del modelo 5D Mohán por medio del semillero de psicología educativa. Los criterios de selección fueron intencionales, siguiendo el criterio de permanencia de los participantes durante los dos años de existencia del semillero. Los estudiantes universitarios eran de quinto y sexto semestre de psicología (seis mujeres y dos hombres, entre los 19 y 22 años), 1 docente de psicología educativa (con experiencia en la implementación del modelo 5D) y 1 estudiante de maestría. La participación de todos era voluntaria y todos hicieron parte, tanto del diseño como de la implementación del proyecto Mohán 5D, siendo estos aspectos diferenciadores frente a otras implementaciones de la 5D, que esencialmente vinculan a estudiantes universitarios en la fase de implementación de la propuesta y en calidad de estudiantes de un curso específico de psicología del desarrollo (Gómez-Estern, MartínezLozano \& Vásquez, 2014). Los participantes firmaron consentimiento informado acerca de su inclusión en la investigación.

\section{Resultados}

\section{Proyecto Mohán 5D como propiciador de transformación de roles}

La implementación del proyecto Mohán 5D posibilitó la construcción de un sistema de actividad diferente al que se desarrolla usualmente en un aula de clase en la que convergen estudiantes y profesores universitarios. Por medio del aprendizaje-servicio que se configura en el modelo 5D, surgen nuevos actores e interacciones que posibilitan el aprendizaje de todos los participantes del sistema. En la figura 2 se plantea una descripción de la conformación del sistema que posibilitó los aprendizajes de los estudiantes universitarios, desde sus propias voces.

Antes de la implementación del Proyecto Mohán 5D, el escenario en el que primordialmente los estudiantes universitarios se formaban para ejercer su profesión era esencialmente el aula universitaria. En este entorno regular de clase, el docente adquiere un $\mathrm{rol}^{3}$ principal como creador de estrategias que posibilitan el aprendizaje de los estudiantes, la relación entre estudiante y docente se caracteriza por una asimetría, y el conocimiento se atribuye en mayor medida al docente (figura 2). En ese sentido, los estudiantes participantes del proyecto 5D plantean diferencias entre el rol que asumían antes de pertenecer al semillero - visión tradicional de la educación en la cual la figura del docente tiene el conocimiento en el aula de clasey el nuevo rol dentro de este espacio.

En el principio, cuando [el semillero] era teórico, pues claramente era más clases-seminario, o sea íbamos a discutir la lectura a partir de lo que se leía [...]. Sin conocimiento base no íbamos a poder hacer nada. Pero ya cuando se convirtió en la práctica, pues cambia totalmente la dinámica de clase $[. .$.$] si vos leías era porque el tema te intere-$ saba, no por la nota. (Participante 1)

La importancia de la motivación por la actividad radica en el carácter voluntario de la intervención de los estudiantes universitarios en el proyecto. De

\footnotetext{
Un rol se entiende como un conjunto de actividades y relaciones que la sociedad espera de una persona que ocupa una posición determinada y que, recíprocamente, implica unas actividades y relaciones de la sociedad para con esa persona (Bronfenbrenner, 1991).
} 


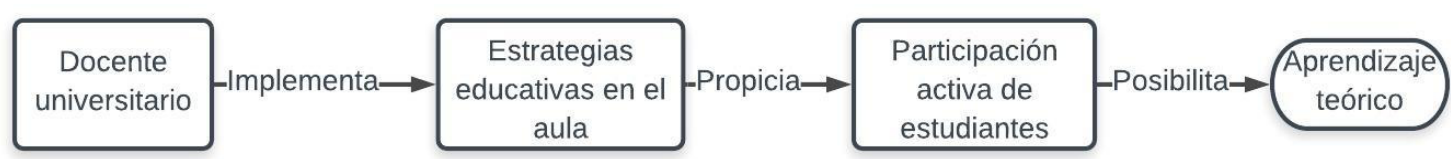

Figura 2. Escenario 1: rol de aprendiz en un aula regular de clase

acuerdo con la participante 1, su presencia en el proyecto no estaba relacionada de forma directa con una nota académica. Los estudiantes se mantuvieron en la actividad, principalmente por el establecimiento de un vínculo con los niños del proyecto y la posibilidad de asumir un rol diferente, no como estudiantes únicamente sino como psicólogos en formación.

[Sobre la motivación al participar] yo siento que en parte se volvió como lo de la Quinta Dimensión, como un microcosmos, entonces uno ya sentía la responsabilidad porque uno ya estaba vinculado con los niños [...] el interés mío [de participar en] una experiencia investigativa, se volvió real cuando yo ya empecé a tener experiencias reales de lo que es ser psicólogo. (Participante 2)

Era un espacio de actividad que el participante 2 describe como un microcosmos, relacionado con lo planteado por Cole $(1999,2017)$ que expresa la creación de un sistema de interacciones que se regulan por principios y valores acordados de forma conjunta, en el que se crean relaciones de carácter dialógico y existe un trato igualitario. En los relatos se observa que se hace una diferenciación entre las interacciones presentes en el proyecto Mohán 5D y aquellas que suceden al interior de un aula regular. Específicamente, se evidencia una importancia dada a los vínculos establecidos entre los estudiantes, y de ellos con los niños participantes de las actividades.

La motivación fue altísima ya cuando tuvimos contacto con los niños, lo de lo voluntario también fue porque era un espacio distinto a las clases, donde no tenés la presión de la nota [...] sino que era un espacio aparte donde, de igual manera, te formas básicamente, pero vas a llevarlo a la acción [...]. También un poco después, cuando pasó a ser más mesa redonda, éramos de psicología del mismo semestre, teníamos lazos de amistad, eso también era motivante porque era un espacio también de encuentro de nosotros. (Participante 3)

Por otra parte, también aparece la importancia del vínculo que se forma entre los estudiantes, el proyecto y los niños y niñas participantes. Se fortalece, por tanto, no solo la amistad entre los estudiantes y participantes, también las relaciones basadas en principios de corresponsabilidad frente a los demás. Al introducirse en este nuevo sistema de actividad, se reconfiguran y amplían los roles $\mathrm{y}$ las actividades que los estudiantes cumplen (y que los docentes cumplen). Así, los estudiantes se involucraron inicialmente en la apropiación teórica de la 5D, posteriormente participaron en el diagnóstico de necesidades realizado participativamente con la ludoteca, seguidamente se adaptó el modelo y se planearon y desarrollaron las actividades con acompañamiento del docente (figura 3). El rol de recepción pasivo de los entornos de educación tradicional fue transformado tanto para los estudiantes, pasando a ser agentes activos, como para el docente quien debió dar lugar a una interacción dialógica en la que se posibilitara la co-construcción de saberes y el desarrollo de competencias. Este nuevo rol se diferencia del aprendiz tradicional en la medida en que articula saberes disciplinares con la práctica supervisada del oficio profesional, y es lo que se ha denominado rol de oficiante. 


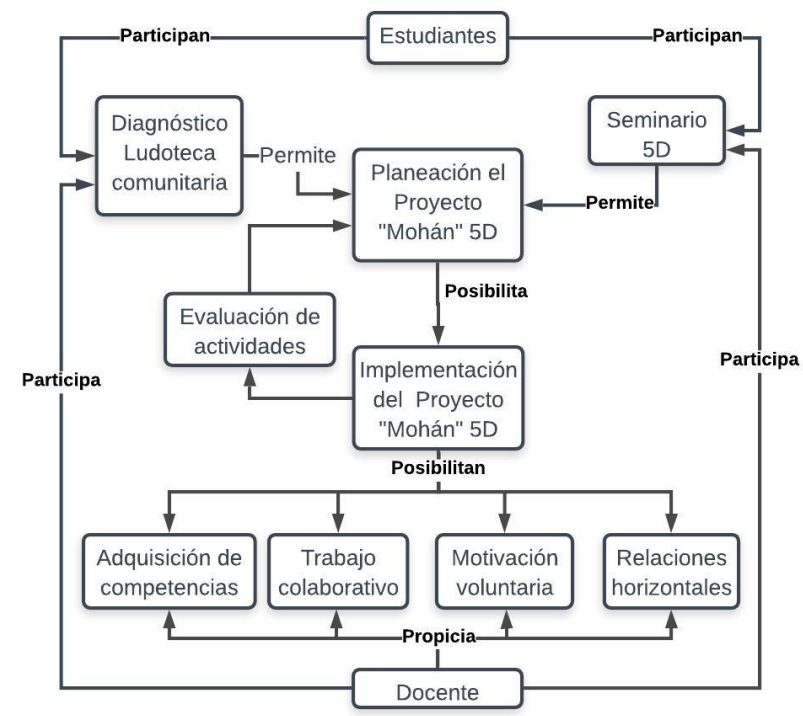

Figura 3. Escenario 2: rol de oficiante por medio del aprendizaje-servicio

Si bien el nuevo rol de oficiante fue adoptado por los estudiantes, la nueva autonomía suponía retos en la distribución de tareas y en la atribución de responsabilidades, proceso que no estaba a cargo del docente, sino que era responsabilidad del colectivo y que inicialmente generó algún grado de desconcierto. Respecto a la distribución de tareas, algunos estudiantes plantearon la dificultad de diferenciar lo que cada una de las personas realizaba al interior de la actividad, porque existía similitud entre las tareas propuestas. Este hecho se corrobora en las actas de reunión que plantean una distribución semejante de tareas. Por ejemplo, en el acta de reunión $\mathrm{N} .^{\circ} 18$ se plantea:

“todos los que han ido a Siloé: adelantar los diarios de campo, todos: hacer las cartas y subirlas mañana jueves en la mañana a drive" (octubre de 2017). "Creo que todos hicimos de todo un poquito [...] entonces para mí no había, así como roles súper establecidos". (Participante 3)

Para propiciar la apropiación del nuevo rol y permitir que los estudiantes asumieran autónomamente las actividades y las formas de relación propias del rol de psicólogos, fue necesario que el docente se ausentara paulatinamente de las jornadas de campo en las cuales se desarrollaban las actividades con los niños y niñas en la ludoteca. La paulatina ausencia del profesor en las actividades de campo significaba la delegación progresiva de la responsabilidad en la ejecución de las actividades en los estudiantes. Lo que no implicaba la ausencia de acompañamiento, ya que la estudiante de maestría participaba de las actividades y cumplía un papel significativo de apoyo. Además, aunque es profesional, era principalmente vista como estudiante, y eso permitía una relación de cercanía con los estudiantes de pregrado.

Al principio [...] todo el mundo estaba como aprendiendo qué era la Quinta Dimensión [...] y luego ya yo creo que el [rol] de todos cambió precisamente porque ya en la parte de acción [el profesor] se ausentaba. Entonces ya todos tuvimos que re organizarnos para poder, por decirlo así, llenar ese vacío de conocimiento que dejaba [El profesor participante del modelo] con lo que nosotros habíamos aprendido y con lo que podríamos hacer. (Participante 2) 
El vacío de conocimiento señalado por el participante 2 fue necesario para que los estudiantes asumieran un rol diferente en su actuar, que también implicó poner en práctica lo ya aprendido. A su vez, la relación que se establece con el docente se modifica, dado que se propicia una relación más horizontal que permite darles voz a los estudiantes durante las jornadas de planeación de las actividades, sin coartarse por la existencia de una calificación.

[Sobre el rol del docente] Uno lo deja de ver cómo un profesor cátedra [...] y lo empieza a ver como un par, lo cual también facilita que nosotros queramos participar, que nosotros podamos hablar con libertad. (Participante 3)

Para llegar al trabajo en campo fue necesario pasar por un momento previo en el que el docente propuso conocer el modelo 5D en una estructura de seminario, en la que era un guía para acceder a material relevante, que posteriormente era discutido en las sesiones tanto del seminario 5D como de planeación e implementación del proyecto.

[Sobre el rol del docente] no tanto el profe que le está enseñando a la gente, sino como de par y él hablaba de su experiencia. O sea, no era como de imponer un conocimiento sino de darnos las lecturas, darnos los autores, y que nosotros a partir de eso propusiéramos ideas. (Participante 1)

Respecto al rol de los estudiantes universitarios, se encuentra que, así como el docente universitario guiaba el actuar de los estudiantes, a su vez ellos se asumían frente a los niños en un rol de mediadores y de guías, tal y como lo expresa la participante 4. Este rol implicaba procesos reflexivos que les permitían preguntarse cómo lograr que los niños por sí mismos resuelvan las actividades propuestas. El rol ya no era por tanto el de estudiante, sino que estaba relacionado con el hacer profesional, ubicando al estudiante como psicólogo en formación. En este sentido, el rol de oficiantes adoptado por los estudiantes significó que en la interacción con los niños durante la ejecución de las actividades se relacionaran como guías o mediadores, a su vez, ellos estaban estableciendo relaciones dialógicas con los niños en la construcción de conocimiento. Las competencias profesionales se expresaron no solo durante la ejecución de actividades, sino que se habían puesto en juego previamente en el diseño y planeación de las actividades de intervención. El proceso de diseño y planeación suponía una relación dialógica entre los estudiantes de pregrado, maestría y docente en el que era relevante la evaluación posterior de las actividades, lo que significaba aprendizajes específicos sobre cada sesión.

Fui una guía en la actividad. Como la idea es que los niños logren llegar al punto final por sí mismos, entonces les daba pautas y ejemplos [...]. Hacía de moderadora en el equipo para que los niños leyeran por turnos el texto. (Participante

4, Diario de Campo)

Así mismo, uno de los principios más relevantes del modelo 5D que se puso en juego fue la creación de un espacio colaborativo en dos niveles: en la interacción establecida con los niños, y en las interacciones al interior del grupo de semillero. Al respecto, la participante 4 destaca la cooperación que se promovía en el primer nivel, de las interacciones con los niños.

Me agradó mucho que entre ellos cooperaran y se ayudaran, aunque a veces uno se adelantaba en el turno del otro y en ocasiones discutían. David se demoraba mucho al momento de hacer las operaciones y entre todos le ayudábamos a resolverlas. (Participante 4, Diario de Campo)

Finalmente, el sistema creado a partir de la 5D tuvo como principales características, en relación con los estudiantes y sus aprendizajes, el 
carácter voluntario de la participación junto al carácter colaborativo, tanto del proyecto como de las relaciones al interior del semillero. La participación voluntaria permitió que los estudiantes se sostuvieran en la actividad a partir de su deseo de hacer una propuesta práctica, relacionarse con los niños y desempeñar actividades propias de un psicólogo. Si bien al interior del semillero sí existían actividades definidas y responsabilidades, la labor del docente no era la de imponer una forma de organización, aspecto que hizo que el semillero se autoorganizara y sus miembros compartieran la responsabilidad de las actividades. Las tareas compartidas facilitaban las relaciones horizontales al interior del grupo. Todos los integrantes compartían un fin común relacionado con llevar a cabo la implementación exitosa del proyecto 5D Mohán.

De esta forma, la configuración de las actividades desarrolladas para la apropiación de la metodología 5D, seminario participativo y la delegación progresiva en la planeación y desarrollo de las actividades de campo permitieron fortalecer el carácter colaborativo del modelo 5D, fortaleciendo el vínculo entre los estudiantes como grupo y entre ellos y los niños. Este aspecto resulta fundamental en tanto permite que el conocimiento se considere como una construcción social, se validen diversos saberes al interior del grupo, y se busque la participación desde diversos campos del conocimiento, así como con niveles diferentes de experticia en el desarrollo de las actividades, para lograr un fin común (Padrós, Sánchez \& Luque, 2012; Sánchez, 2018). En este punto, el aprendizaje se da en todos los integrantes que interactúan en el semillero. De forma específica, la colaboración entre los diferentes integrantes del semillero desde la asunción de roles horizontales posibilitó el cambio en los roles de los estudiantes de psicología: participaban tanto en el desarrollo de la actividad, como en su evaluación y en la planeación de actividades subsiguientes.

\section{Aprendizajes de los estudiantes participantes del semillero}

\section{Aprendizajes para el oficio}

Dentro de esta categoría de aprendizaje para el oficio, se retoma a Senett (2009) — para quien "la gente puede aprender de sí misma a través de las cosas que produce" (p. 12) - para mostrar los aprendizajes de los estudiantes universitarios por medio de los productos de la planeación de actividades del proyecto Mohán 5D y de sus propias voces.

Al respecto, uno de los componentes que destacaron los estudiantes de la implementación del modelo 5D fue el uso de cartas entre Mohán y los niños de la ludoteca. En relación con la relevancia dada por los estudiantes al uso de las cartas, se señalaron dos aspectos: 1) se resalta la potencialidad de la narración como herramienta del trabajo del psicólogo, 2) el carácter significativo del aprendizaje alcanzado por los estudiantes.

Lo más significativo fue poder observar cómo, a través de la imaginación, del cuento, de la historia, uno puede llegarles a los niños de una manera [...] que ellos mismos entren a algo que les guste y los motive. (Participante 1)

Uno de los objetivos al construir el proyecto Mohán en relación con los estudiantes era permitir que ellos se apropiaran de herramientas conceptuales y metodológicas que pudieran usar posteriormente en su intervención como psicólogos. Dentro de los elementos teóricos más relevantes al respecto se encontraba la concepción del Yo como producto de una narrativa resultante de una mezcla entre los relatos propios y los relatos de otros (Bruner, 2002). En relación con lo anterior, el modelo 5D establece dentro de sus principios la narratividad, pues "el relato es una forma de organización del sentido universal, presente a todas 
las culturas, que puede ser utilizado como vía de acceso a la práctica escolar" (Padrós, Sánches-Búsques, Lalueza, Crespo \& Lamas, 2014, p. 154). La narrativa del Proyecto Mohán 5D gira alrededor de la historia de Mohán, de la creación del espacio o club de amigos del Mohán y del intercambio comunicativo y personalizado por medio de cartas entre el personaje mágico (llevado a cabo por los integrantes del semillero de psicología) y los niños participantes. Las cartas son, en este orden de ideas, artefactos mediadores (Cole, 1999) en los que se ponen en juego tanto aspectos narrativos que encuadran y dan sentido a la experiencia de los participantes, como aprendizajes específicos relativos de la lectura y la escritura.

Uno se imagina que llega, los saluda [a los niños], ellos hacen caso, les propones la actividad... y muchas veces no [funciona]. Entonces al nosotros chocar con eso tuvimos que buscar la forma de llegarles [...], cuando había desorden, el hecho de que hubiera una carta y que se tuvieran que sentar porque mi carta llegó [como respuesta] a lo que yo le escribí específicamente eso los calmaba. Y que fuera Mohán el que siguiera escribiendo. (Participante 3)

Las cartas, junto a otras herramientas como las guías de las actividades (retos), se organizaban mediante una narrativa (en donde Mohán era central) que tenía sentido, ya que estaba orientada a metas determinadas y se vinculaba con los intereses de todos los integrantes, pues permitía incluir a los niños en la actividad de acuerdo con sus propios intereses. Así, los estudiantes de psicología vivenciaron la potencialidad del uso de narrativas en el trabajo con niños para motivarlos hacia labores escolares y exploraron cómo la comunicación efectiva que proponía la carta resultaba estimulante para el aprendizaje de la lectura y la escritura que estaban afrontado los niños y niñas participantes.
Cuando era el momento de las cartas todo se calmaba, las actividades eran un caos, no malo, sino que como que todos estaban disfrutando la actividad y los niños son bulliciosos [...]. Pero cuando ellos están en el momento de las cartas y traemos el buzón, ellos [los niños]: “¿y qué me dijo el mohán?” “¿Y qué le voy a responder?”, entonces claro cuando nosotros veíamos esa intención entonces dijimos tenemos que responderles de igual manera. (Participante 6)

La narrativa de la 5D Mohán permitió también la creación de vínculos entre los niños y los estudiantes participantes. Estos últimos asumieron con responsabilidad su papel de responder a lo pactado al interior de la comunidad 5D, específicamente, asumieron el papel de Mohán.

Por otra parte, en el proceso resultó relevante que los aprendizajes fueran significativos para los estudiantes. Se conoce que el modelo 5D propicia el aprendizaje significativo frente al aprendizaje por transmisión de conocimientos, por medio de la participación y el trabajo colaborativo orientado al cumplimiento de metas de los participantes de la experiencia (Padrós, et al. 2014; Jiménez, Lalueza \& Fardella, 2017). Por aprendizaje significativo se entiende, siguiendo a Coll (1988), aquel aprendizaje que permite la construcción de relaciones entre los nuevos conocimientos y lo ya conocido. En este sentido, el proyecto Mohán 5D permitía establecer relaciones fuertes entre los conocimientos teóricos de la psicología cultural y las prácticas fundamentadas en dicha teoría. Un ejemplo de ello es la producción escrita de cartas (como artefacto cultural que materializa la narrativa propuesta entre el personaje y los niños participantes) y las referencias a estas como aspectos significativos del proceso vivido por los estudiantes participantes (como lo plantea el participante 5). Es necesario resaltar que el aprendizaje significativo requiere de un alto grado de responsabilidad de parte de los estudiantes, pues 
uno de sus componentes es la intencionalidad o actitud favorable que el estudiante tome respecto a su proceso de aprendizaje (Coll, 1988).

[Durante la actividad número 4] Me pareció interesante el hecho de que lograran identificar las emociones y plasmarlas en un dibujo. [...] dentro del grupo hubo una pelea y el niño (Darwin) lo plasmó en su dibujo y además demandó al Mohán herramientas para solucionar el conflicto por medio de la carta. (Diario de campo, Participante 5)

La participación y apropiación de las prácticas y el sentido otorgado a los artefactos, como las cartas, dan cuenta de la apropiación del modelo 5D por parte de los estudiantes universitarios, asumiéndolo como una metodología alternativa orientada bajo conceptos de la psicología cultural. De este modo, el aprendizaje colaborativo se refrenda no solo como propuesta hacia los niños en las actividades del proyecto, sino que la cooperatividad se evidenció además en el desarrollo semanal de las planeaciones del semillero. Los estudiantes se reconocieron a sí mismos como mediadores, propiciando a su vez la motivación y apropiación de los artefactos y los principios del modelo por parte de los niños.

Me agradó mucho que entre ellos cooperaran y se ayudaran, aunque a veces uno se adelantaba en el turno del otro y en ocasiones discutían. Darwin se quedaba mucho al momento de hacer las operaciones y entre todos le ayudábamos a resolverlas (Diario de campo, Participante 4).

La 5D como son juegos, son cosas colectivas, consensuadas [...] uno les puede estar enseñando matemáticas y están aprendiendo al final de cada reto. (Participante 2)

Este tipo de herramientas de trabajo para los psicólogos, junto con el entendimiento de los alcances de las herramientas, resultan relevantes en entornos actuales que se caracterizan por su complejidad, que hacen necesarias las competencias de aprendizaje colaborativo y el uso de redes sociales que implican el entendimiento del conocimiento como una configuración dialógica (Yamazumi, 2006 en Sánchez, 2018).

\section{Aprendizajes personales: competencias}

Los estudiantes ubican aprendizajes de competencias profesionales como trabajo en equipo, liderazgo, manejo de grupo y responsabilidad. Las interacciones al interior del grupo del semillero fueron decisivas, en estos encuentros requerían poner en acción dichas competencias. Lo anterior guarda semejanzas con el pensamiento de Dewey que plantea " [...] en una buena escuela, los alumnos aprenden a ser ciudadanos realizando proyectos en común con sus compañeros y resolviéndolos en conjunto, con un espíritu respetuoso, pero a la vez crítico" (Citado por Nussbaum, 2010, p. 97). Al respecto, la participante 3 plantea:

[Participar en la experiencia] posibilitó aprender muchísimas cosas y más que teóricas de competencias de trabajo en equipo, de liderazgo, algunas veces de manejo de grupo [...] trabajo en equipo: a pesar de que todos éramos de psicología y coincidíamos la mayoría en los semestres, teníamos a veces visiones distintas de lo que se podía hacer con los niños o teníamos propuestas distintas para las actividades [...]. Trabajo en equipo más que todo en las actividades para negociar un poco que vamos a hacer [...], finalmente todos aportamos desde lo que habíamos leído, desde lo que habíamos vivido en la visita con los niños, eso me parece una competencia porque vamos a tener choques a veces, entonces saber manejar esos choques para saber qué íbamos a hacer en la próxima actividad pues me pareció importante. (Participante 3)

En el relato anterior de la participante 3, se presentan los componentes de la definición de competencia: capacidad de un individuo para 
cumplir exitosamente, por medio de su actuar o de sus elecciones en contextos particulares, demandas de compleja resolución (Rychen \& Salganik, 2003, en Lozano et al., 2012). En primer lugar, los estudiantes se encuentran en dos contextos particulares: por una parte, los momentos de interacción con otros estudiantes universitarios, con el docente y la estudiante posgraduada durante el seminario 5D y la planeación de las actividades del proyecto en la universidad y la evaluación posterior de cada actividad. Por otra parte, los momentos de encuentro con los niños durante la implementación del proyecto 5D.

En segundo lugar, de acuerdo con la participante 3 , los estudiantes universitarios se encuentran con demandas complejas en dichos contextos, cuyo carácter de complejidad se desliga de diversas características como la interacción con otros que tienen visiones diferentes pero que tienen como meta llegar a un consenso frente a la planeación de una actividad.

En tercer lugar, se ubican recursos propios de la participante 3 para afrontar las diferentes demandas de los contextos específicos, que tienen en cuenta tanto procesos cognitivos como procesos no cognitivos, que implicaron en su caso la negociación de tareas al interior del grupo para llevar a término las actividades del proyecto. "Uno ya tenía una responsabilidad con, porque es que uno ya sabía que uno tenía deseos de niños en las cartas" (Participante 2).

Siendo esta una actividad de carácter voluntario, el compromiso ya no está mediado por la obtención de una nota, como es habitual en las tareas escolares, sino por el compromiso adquirido con los niños y niñas, y con la problemática social reconocida y diagnosticada conjuntamente con la comunidad. Los estudiantes logran descentrar sus intereses de sí mismos e involucrarse de manera comprometida y responsable con un objetivo establecido por ellos mismos, al tiempo que cumplen una función que los ubica en el ejercicio del oficio de un psicólogo educativo: aprenden a realizar un diagnóstico, reconocen las necesidades y demandas de la comunidad, establecen propósitos para la intervención de manera participativa y planean y desarrollan las actividades haciendo uso de los artefactos que propone el modelo 5D, las cartas como artefacto narrativo que media entre los niños y el personaje mágico, actividades lúdico-educativas, con diversos niveles de desempeño que motivan a los niños al tiempo que, por medio de la interacción con los niños, van descubriendo cuándo y cómo intervenir, por ejemplo, para obtener la atención del grupo.

Finalmente, en la siguiente reflexión del participante 2, se resumen las posibilidades que brinda el proyecto 5D para el aprendizaje del oficio y de las competencias.

(Aprendizaje del semillero) pues con lo de ser psicólogo [...], esas pequeñas situaciones, más o menos controladas, que le van dando a uno [la posibilidad] de estar como en un rol de psicólogo, incluso dentro del salón, porque, por ejemplo, cuando uno está diseñando, uno está teniendo discusiones con los compañeros. Ya es un espacio en el que uno tiene que tomar una posición [...], uno se va llenando de unos argumentos chéveres o sea que responden a conceptos, teorías, pero también a propósitos. (Participante 2)

Cuando el participante 2 expresa la existencia de "pequeñas situaciones más o menos controladas" se ubica el diseño metodológico de la experiencia, que fue definido por Cole (2016) como un experimento por diseño que posibilita la creación de diferentes situaciones promotoras del desarrollo o, en palabras de Bronfenbrenner (1991), situaciones que les permiten a los estudiantes universitarios hacer una transición ecológica y asumir un nuevo rol como psicólogos. A su vez, el nuevo rol de psicólogos implica poner en juego unas habilidades específicas de planeación y lograr no solo conectar la teoría con la práctica profesional, sino llegar a pensar con la teoría e incluso aprender de 
sí mismos por medio de lo que producen, siguiendo a Senett (2009). Finalmente, se plantea que el aprendizaje es significativo en el sentido en que las actividades diseñadas no solo responden a la teoría o a los conceptos aprendidos, sino a propósitos definidos previamente para estas, de acuerdo con el participante 2, es decir que los elementos prácticos y teóricos permiten a los estudiantes la construcción de significados, al tiempo que van desarrollando habilidades propias de su oficio profesional como psicólogos.

\section{Discusión}

A pesar de los esfuerzos por transformar las concepciones y las prácticas educativas en los entornos escolares, aún hoy estas se basan principalmente en "una epistemología objetivista y una visión de sentido común de la enseñanza y del aprendizaje como transmisión de conocimiento" (Tynjälä, 2001, p. 2). En el estudio que hacen Marton, Dall'Alba y Beaty (1993; en: Tynjälä, 2001), acerca de las concepciones sobre el aprendizaje que tienen estudiantes universitarios, establecen dos grupos en sus hallazgos: 1) El aprendizaje se relaciona con "adquirir algo ya hecho", lo que supone el almacenamiento en la memoria de un producto externo producido por "otro". 2) El aprendizaje se entiende como una "transformación", aprender está relacionado con obtener un significado. De acuerdo con esto, dice Tynjälä (2001) "cambiar como persona es desarrollar estructuras de significado completamente nuevas para toda la vida" (p. 2).

En el desarrollo del proyecto Mohan 5D, se encuentran evidencias tanto en los productos como en la ejecución misma de las actividades, del aprendizaje obtenido por los estudiantes.

De forma específica, el aprendizaje de los estudiantes universitarios, cuando se tienen como referencia sus voces, da cuenta no solo de la apropiación del modelo 5D (uso de conceptos de la psicología cultural), sino de la transformación del rol de los estudiantes participantes: pasan del rol de estudiantes al de oficiantes. En este sentido, los hallazgos evidencian la apropiación de competencias propias del oficio como psicólogos.

Este nuevo rol de oficiantes implica la capacidad de dar cuenta de competencias específicas de un oficio particular por medio de la participación en la ejecución de las actividades propias del oficio, sin ser aún expertos. El rol que se propone generalmente a los estudiantes es el de aprendices, por el cual se les evalúa, en consecuencia, no se les exigen las competencias de la profesión sino principalmente las del oficio de estudiante, estas competencias se refieren principalmente a actividades de orden académico. Aunque establecer el vínculo entre lo que se les enseña en abstracto y la práctica profesional conlleva dificultades.

La transformación del rol de estudiante al de oficiante es una forma de propiciar los aprendizajes necesarios para el oficio, aunque este pasaje solo sea posible cuando se proponen y usan metodologías distintas a la transmisión oral que prevalece en la educación universitaria. Lo anterior implica una transformación del rol docente, por ejemplo, en la delegación progresiva de las actividades en los estudiantes, lo que supone ofrecer confianza al tiempo que los recursos necesarios para que los estudiantes logren adelantar una actividad educativa ética y responsable. Así, la labor del docente debe configurar un espacio de retroalimentación y evaluación permanente de la actividad, su rol es el de una guía experta que orienta y cuestiona sin ofrecer una actitud directiva. Este cambio es el que permite a los estudiantes ocupar el espacio vacío que deja el docente y llenarlo con su nuevo rol. Por lo anterior, el docente es percibido como un par y no como quien da cátedra.

En este sentido, la participación de la estudiante de posgrado resultó en un apoyo valioso, en tanto que permitió configurar otra zona de desarrollo proximal con los estudiantes universitarios, siendo soporte para la evaluación y planeación de las actividades de campo. 
Por otra parte, el aprendizaje-servicio, y en particular el desarrollo del proyecto Mohán 5D, aporta en el desarrollo de las competencias necesarias para su ejecución, en la medida que provee a los estudiantes los recursos necesarios para afrontar las dificultades propias de la intervención en un contexto real, al tiempo que ofrece un entorno colaborativo para la resolución de problemas y para el aprendizaje, lo cual ha sido corroborado en otros estudios como el de Gómez-Estern, Martínez-Lozano y Vásquez (2014), y García-Romero, Sánchez, y Lalueza (2018).

Esta idea de la puesta en marcha de competencias en situaciones de compleja resolución por parte del estudiantado resulta coherente con el paradigma constructivista, en tanto se le da una prioridad al contexto en el cual se llevan a cabo los aprendizajes y el docente asume un rol de acompañamiento de los estudiantes en la construcción de significados por medio de interacciones en diversas actividades (Serrano \& Pons, 2011).

Finalmente, el modelo 5D permite con claridad establecer un entorno colaborativo no solo para el aprendizaje de los estudiantes universitarios, sino para el de los niños, niñas y las comunidades donde se desarrolla, en tanto que brinda una opción de resolución y afrontamiento a las demandas y necesidades que presentan, siendo justamente este factor una de las características que permitió el establecimiento de un vínculo fuerte de los estudiantes universitarios con el proyecto y que posibilitó la configuración de un espacio polifónico en donde las voces de niños y niñas tenían un lugar, y en el que el sistema de actividad permitió articular los intereses de diversos actores, lo que resulta poco habitual en los espacios educativos tradicionales en donde solo la voz del docente tiene especial importancia.

\section{Referencias}

Baquero, R. (1996). Vigotsky y el aprendizaje escolar (Vol. 4). Buenos Aires: Aique.
Blanton, W., Bremme, D., \& Nocon, H. (2006). The Effects of Fifth Dimension Participation on Undergraduates. In M. Cole \& DLC. The Fifth Dimension. An After-School Program Built in Diversity (pp. 129-159). New York: The Distributed Literacy Consortium.

Bronfenbrenner, U. (1991). La ecología del desarrollo humano. Paidos Ibérica.

Bruner, J. (2002). La fábrica de hacer historias. Buenos Aires: FCE.

Cole, M. (1999). Psicología cultural. Una disciplina del pasado y del futuro. Madrid: Ediciones Morata.

Cole, M. (2014). Towards a mesogenetic methodology. In A. Blunden (Eds.). Collaborative projects: An interdisciplinary study (pp. 361-367). Leiden, The Netherlands: Brill.

Cole, M. (2016). Designing for development: Across the scales of time. Developmental. psychology, 52(11), 1679-1689. Doi: http://dx.doi.org/10.1037/ $\operatorname{dev} 0000156$

Cole, M. (2017). Idiocultural design as a tool of cultural psychology. Perspectives on Psychological Science, 12(5), 772-781.

Coll, C. (1988). Significado y sentido en el aprendizaje escolar. Reflexiones en torno al concepto de aprendizaje significativo. Infancia y aprendizaje, 11(41), 131-142.

Fardella, C., \& Carvajal Muñoz, F. (2018). Los estudios sociales de la práctica y la práctica como unidad de estudio. Psicoperspectivas, 17(1). Doi: https://doi.org/10.5027/psicoperspectivas-vol17- issue 1-fulltext-1241

García-Romero, D., Sànchez-Busqués, S., \& Lalueza-Sazatornil, J. L. (2018). Exploring the Value of Service Learning: Students' assessments of Personal, Procedural and Content Learning. Estudios sobre Educación, 35, 557-577.

Gómez-Estern, B., Martinez-Lozano, V., \& Vasquez, O. (2014). Real Learning in Service Learning: Lessons from La Clase Magica in the US and Spain. International Journal for Research on Extended Education, 2(2), 63-78. Doi:10.3224/ ijree.v2i2.19547 
Gutiérrez, K. D., \& Jurow, A. S. (2016). Social design experiments: Toward equity by design. Journal of the Learning Sciences, 25(4), 565-598, Doi: 10.1080/10508406.2016.1204548

Jiménez, F., Lalueza, J. L., \& Fardella, K. (2017). Aprendizajes, inclusión y justicia social en entornos educativos multiculturales. Revista Electrónica de Investigación Educativa, 19(3), 10-23. Doi: https://doi.org/10.24320/redie.2017.19.3.830

Lamas, M., Sánchez-Busqués, S., \& Lalueza, J. L. (2018). Changes in school through a long-term project of action research. Building bridges between school practice and Roma communities. Educational Action Research. Doi: 10.1080/09650792.2018.1509721

Lalueza, J. L., Sánchez-Busqués, S., \& Padrós, M. (2016). Creando vínculos entre universidad y comunidad: el proyecto Shere Rom, una experiencia de aprendizaje servicio en la Facultad de Psicología de la Universitat Autònoma de Barcelona. RIDAS, Revista Iberoamericana de Aprendizaje Servicio, 2, 33-69. Doi: 10.1344/ RIDAS2016.2.3

Lozano, J. F., Boni, A., Peris, J., \& Hueso, A. (2012). Competencies in Higher Education: A Critical Analysis from the Capabilities Approach. Journal of Philosophy of Education, 46(1), 132-147. Doi: http://dx.doi.org/10.1111/j.14679752.2011.00839.x

Menand, L. (2001). The Metaphysical Club: A story of ideas in America. Nueva York: Farrar, Straus, and Giroux.

Nussbaum, M.C. (2010). Sin fines de lucro. Por qué la democracia necesita de las humanidades. Madrid: Katz.

Padrós M., Sánchez S., \& Luque M. J. (2012). Shere Rom: creando una microcultura para la inclusión socioeducativa. Quaderns de Psicología, 14(2), 87-99.

Recibido: agosto 28, 2019

Aprobado: septiembre 17, 2019
Padrós, M., Sánchez-Busqués, S., Lalueza, J. L., Crespo, I., \& Lamas, M. (2014). El proyecto Shere Rom. Fundamentos de una comunidad de prácticas para la inclusión educativa. Psicología, Conocimiento y Sociedad, 4(2), 138-162.

Pozo, J. I., \& Monereo, C. (2009). Psicología del aprendizaje universitario. Madrid: Ediciones Morata.

Sánchez, J. E. (2018). Características e implicaciones relacionales de dos propuestas innovadoras en educación. En O. Bravo (Ed.). Una década del programa de psicología en la Universidad Icesi (pp. 59-77). Cali: Editorial Icesi.

Senett, R. (2009). El artesano. Barcelona: Editorial Anagrama.

Serrano González-Tejero, J. M., \& Pons Parra, R. M. (2011). El constructivismo hoy: enfoques constructivistas en educación. Revista electrónica de investigación educativa, 13(1), 1-27. Tynjälä, P. (2001). Wrinting as a learninig tool. Symposium "International Research into Writing: an European- American Dialogue on Global and Local Issues"; AREA, 4-12. 82th Annual Meeting of the American Educational Research Association.

Unesco. (2016). Incheon Declaration and Framework for Action for the implementation of Sustainable Development Goal 4. Recuperado de http:// uis.unesco.org/sites/default/files/documents/ education-2030-incheon-framework-for-actionimplementation-of-sdg4-2016-en_2.pdf

Vygotski, L. S. (1993). Pensamiento y lenguaje. Obras escogidas, tomo 2 (pp. 9-348). [Trabajo original publicado 1934]. Madrid: Visor.

Wenger, E. (2008). Communities of practice: Learning, meaning, and identity. Nueva York: Cambridge University Press. Doi: http://dx.doi. org/10.1017/CBO9780511803932 